\title{
Estimating the Mortality of Patients With Severe Aortic Stenosis Who Develop Heart Failure
}

\author{
Kyung-Duk Min, MD, PhD
}

$\mathbf{T}$ he worldwide prevalence of aortic stenosis (AS), the most common valvular disease in the elderly, is increasing as the average lifespan increases. Approximately $2 \%$ of people aged more than 65 years and $4 \%$ of people aged more than 85 years are affected with AS. ${ }^{1}$ The development and early progression of AS is often asymptomatic, but is followed by a symptomatic phase associated with high mortality. ${ }^{2-4}$ Heart failure is the most serious comorbidity for patients with severe AS, ${ }^{2}$ and once it develops the median life expectancy is approximately 2 years. Despite the poor prognosis, there is no medical therapy to treat AS or to reduce the mortality rate. The treatment of choice for patients with symptomatic AS is aortic valve replacement (AVR). Additionally, in the past decade, transcatheter AVR (TAVR) has gained popularity as a therapeutic option for high-risk patients who are not eligible for open AVR surgery. However, even in the latest guideline for valvular heart disease published by the AHA/ ACC, TAVR is not recommended for patients with a life expectancy $<1$ year or for patients with a chance of "survival with benefit" of $<25 \%$ at 2 years. ${ }^{5}$ Therefore, the risk stratification of patients with symptomatic AS is an issue of great concern.

\section{Article p 2455}

In this issue of the Journal, Kawase et $\mathrm{al}^{6}$ report on the effect of low systolic blood pressure (SBP) on the prognosis of patients with acute decompensated heart failure (ADHF) because of moderate-to-severe AS. They retrospectively enrolled 107 ADHF patients with moderate-to-severe AS (maximal jet velocity $\geq 3 \mathrm{~m} / \mathrm{s}$ or aortic valve area $<1.5 \mathrm{~cm}^{2}$ ). After excluding patients with acute coronary syndrome or AVR and those lost to follow-up, 71 patients were included in the study. The primary endpoint was 1-year mortality and the authors compared clinical parameters between patients who had died at 1 year and those who survived. They demonstrated that the SBP at admission, the estimated glomerular filtration rate, and the proportion of patients with a left ventricular ejection fraction (LVEF) $<50 \%$ were significantly different between patients who survived and those who died. Also, they showed that a low admission SBP $(<120 \mathrm{mmHg})$ independently predicted a lower mortality rate at 1 year (adjusted hazard ratio, 2.41; $95 \%$ confidence interval, 1.04-5.57; $\mathrm{P}=0.033$ ).

Low SBP has been identified as a predictor of poor prognosis in patients with HF. The Acute Decompensated Heart Failure National Registry (ADHERE) investigators reported that low admission SBP $(<115 \mathrm{mmHg})$ was the second independent predictor for in-hospital mortality among 39 variables, although valvular diseases were not included. ${ }^{7}$ The Organized Program to Initiate Lifesaving Treatment in Hospitalized Patients with Heart Failure (OPTIMIZE-HF) investigators evaluated the association between quartiles of admission SBP and outcomes in hospitalized ADHF patients. They demonstrated that lower admission SBP $(<120 \mathrm{mmHg})$ was related to a higher in-hospital mortality rate and a higher post-discharge mortality rate $(7.2 \%$ and $14.0 \%$, respectively) compared with higher admission SBP. Although both studies showed that lower admission SBP predicted poorer outcome, the mortality of patients with ADHF secondary to AS was not specifically evaluated. Therefore, the present study has high clinical importance.

What is the pathophysiological effect of low SBP in patients with severe AS? Severe AS is usually defined as a valve area $\leq 1.0 \mathrm{~cm}^{2}$ with an aortic velocity $<4 \mathrm{~m} / \mathrm{s}$, which corresponds to a mean pressure gradient $\leq 40 \mathrm{mmHg}$. However, it is increasingly recognized that a substantial number of patients classified with severe AS have lower pressure gradients, despite a narrowed aortic orifice. This entity has been widely accepted as low-flow, low-gradient (LF-LG) AS. In fact, the latest AHA/ACC guidelines classified symptomatic AS into 3 categories: D1 for symptomatic severe high-gradient AS, D2 for symptomatic severe LF-LG AS with reduced LVEF, and D3 for symptomatic severe LF-LG AS with normal LVEF or paradoxical low-flow severe AS, typically accompanied by concentric left ventricular (LV) hypertrophy with a small LV cavity (Figure). ${ }^{8,9}$ In this context, given that vascular resistance does not vary, low SBP is thought to be the consequence of one of the following scenarios: (1) high or average gradient with decreased intraventricular pressure, (2) low gradient with preserved intraventricular pressure, or (3) low gradient with decreased intraventricular pressure. Generally, sympathetic nerve activity is upregulated in ADHF patients, resulting in high vascular resistance, and SBP remains normal or high under substantial stroke volume. Therefore, in scenario (1), LV function is thought to be impaired and unable to generate sufficient stroke volume. This scenario may be involved in stage D1 with reduced LVEF. In scenarios (2) and (3), it is thought that the $\mathrm{LV}$ fails to generate enough stroke volume despite the low gradient, indicating LV dysfunction. These scenarios would be classified as stage D2 or D3. In any case, these scenarios may be the consequence of systolic dysfunction, which is an obvious predictor of poor prognosis. ${ }^{10}$ Although further studies are required, these pathophysiological models may explain the high mortality rate in ADHF patients with low admission SBP.

The opinions expressed in this article are not necessarily those of the editors or of the Japanese Circulation Society.

Received August 25, 2014; accepted August 25, 2014; released online September 16, 2014

Department of Clinical Research and Development, and Department of Cell Biology, National Cerebral and Cardiovascular Center, Suita, Japan

Mailing address: Kyung-Duk Min, MD, PhD, Department of Clinical Research and Development, National Cerebral and Cardiovascular

Center, 5-7-1 Fujishirodai, Suita 565-8565, Japan. E-mail: min@ncvc.go.jp

ISSN-1346-9843 doi:10.1253/circj.CJ-14-0949

All rights are reserved to the Japanese Circulation Society. For permissions, please e-mail: cj@j-circ.or.jp 


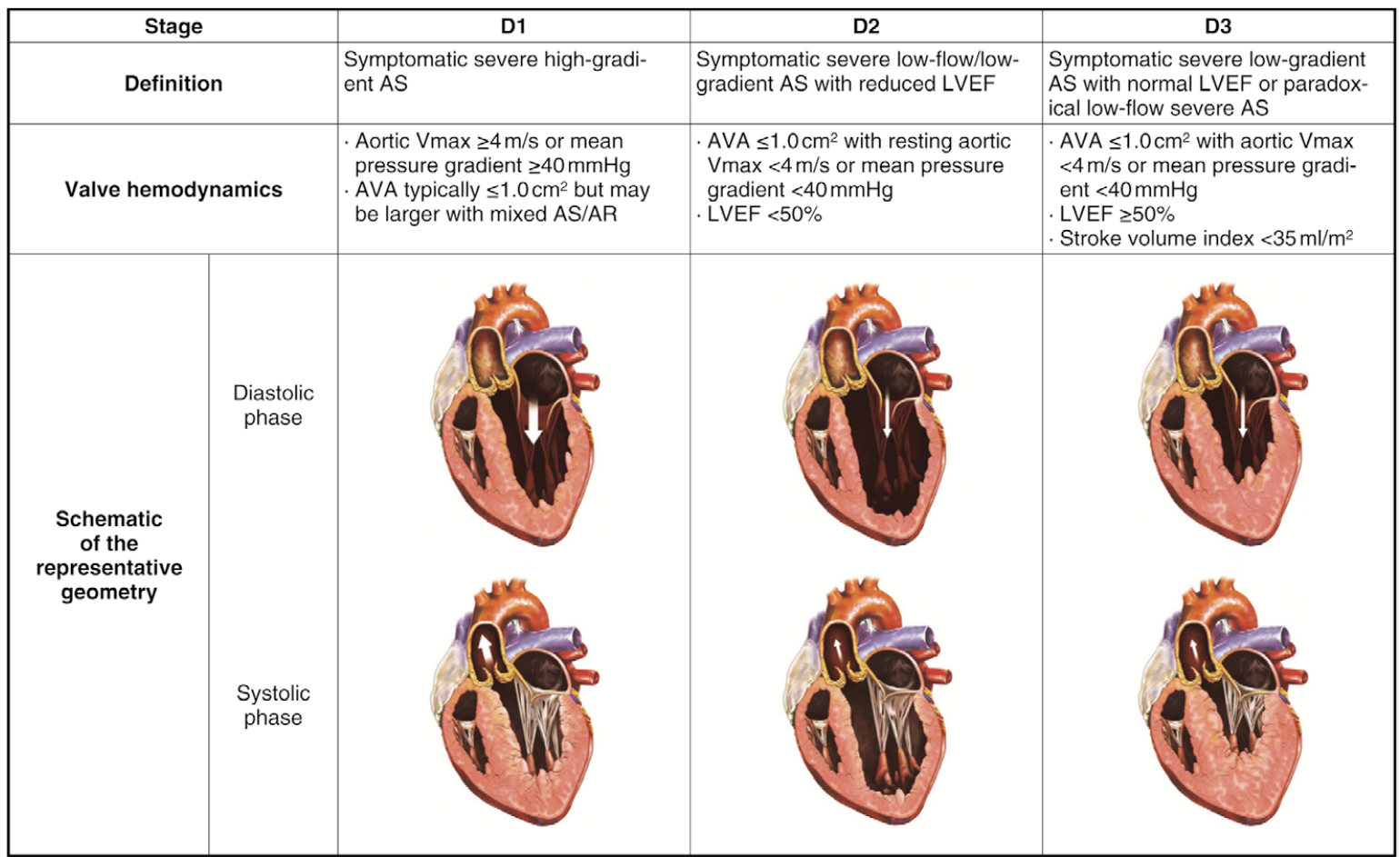

Figure. Classification of symptomatic severe AS and schematic of the representative geometries. Symptomatic severe AS is defined as stage $D$ in the latest version of the AHA/ACC guidelines for valvular heart disease. The table was adapted with permission from Nishimura RA et al. ${ }^{5}$ The scheme was arranged based on Pibarot and Dumesnil. ${ }^{9}$ AR, aortic regurgitation; AS, aortic stenosis; AVA, aortic valve area; LVEF, left ventricular ejection fraction.

Another interesting finding of the present study is that the SBP at discharge was not significantly different between patients who survived and those who died (117 \pm 19 vs. $110 \pm$ $18 \mathrm{mmHg}, \mathrm{P}=0.15)$, whereas the admission SBP was significantly higher in patients who survived than in those who died $(152 \pm 43 \mathrm{mmHg}$ vs. $116 \pm 32 \mathrm{mmHg}, \mathrm{P}<0.001)$. This finding indicates that the contractile reserve at admission, probably provoked by the sympathetic storm in ADHF, was higher among the surviving population than among the dead population. This is consistent with the clinical evidence that patients with a higher contractile reserve on a dobutamine stress test show better outcomes. ${ }^{11}$

Despite the limitations of the retrospective study design, the present study provides important information on the prediction of the outcome of patients with ADHF secondary to severe AS. However, some questions remain. The present study included patients with moderate AS, who are not generally considered for surgical therapy. In future studies, populations should be analyzed based on the severity of AS. In addition, further research is needed to determine whether the findings of the present study also apply to younger patients with AS.

We are now facing a new era with numerous therapeutic choices for severe AS. Risk stratification is essential to ensure the best possible outcome for each patient.

\section{References}

1. Stewart BF, Siscovick D, Lind BK, Gardin JM, Gottdiener JS, Smith VE, et al. Cardiovascular health study. J Am Coll Cardiol 1997; 29: 630-634.

2. Ross J Jr, Braunwald E. Aortic stenosis. Circulation 1968; 38: 61-67.
3. Kelly TA, Rothbart RM, Cooper CM, Kaiser DL, Smucker ML, Gibson RS. Comparison of outcome of asymptomatic to symptomatic patients older than 20 years of age with valvular aortic stenosis. Am J Cardiol 1988; 61: 123-130.

4. Turina J, Hess O, Sepulcri F, Krayenbuehl HP. Spontaneous course of aortic valve disease. Eur Heart J 1987; 8: 471-483.

5. Nishimura RA, Otto CM, Bonow RO, Carabello BA, Erwin JP 3rd, Guyton RA, et al. 2014 AHA/ACC guideline for the management of patients with valvular heart disease: A report of the American College of Cardiology/American Heart Association Task Force on Practice Guidelines. J Am Coll Cardiol 2014; 63: e57-e185, doi:10. 1016/j.jacc.2014.02.536.

6. Kawase Y, Kadota K, Nakamura M, Tada T, Hata R, Miyawaki H, et al. Low systolic blood pressure on admission predicts mortality in patients with acute decompensated heart failure due to moderate to severe aortic stenosis. Circ J 2014; 78: 2455-2459.

7. Fonarow GC, Adams KF Jr, Abraham WT, Yancy CW, Boscardin WJ. Risk stratification for in-hospital mortality in acutely decompensated heart failure: Classification and regression tree analysis. JAMA 2005; 293: $572-580$.

8. Gheorghiade M, Abraham WT, Albert NM, Greenberg BH, O'Connor CM, She L, et al. Systolic blood pressure at admission, clinical characteristics, and outcomes in patients hospitalized with acute heart failure. JAMA 2006; 296: 2217-2226.

9. Pibarot P, Dumesnil JG. Low-flow, low-gradient aortic stenosis with normal and depressed left ventricular ejection fraction. $J$ Am Coll Cardiol 2012; 60: 1845-1853.

10. Shibayama K, Watanabe H, Tabata M, Sasaki S, Fukui T, Umemura $\mathrm{J}$, et al. Impact of ejection fraction on long-term outcome after elective aortic valve replacement in octogenarians with aortic stenosis. Circ J 2012; 76: 1761 - 1767.

11. Nishimura RA, Grantham JA, Connolly HM, Schaff HV, Higano ST, Holmes DR Jr. Low-output, low-gradient aortic stenosis in patients with depressed left ventricular systolic function: The clinical utility of the dobutamine challenge in the catheterization laboratory. Circulation 2002; 106: 809-813. 